\title{
Correlation between Serotonin Transporter Promoter Gene Polymorphism and PTSD in Children
}

\author{
Juncheng Guo', Min Guo ${ }^{2}$, Ping Huang33, Yijun Yang', Xiangling Jiang², Weidong Cen ${ }^{4}$ \\ ${ }^{1}$ Central South University, Xiangya School of Medical Affiliated Haikou Hospital, Haikou, China \\ ${ }^{2}$ Department of Psychology, Hainan General Hospital, Haikou, China \\ ${ }^{3}$ University of South China, Hengyang, China \\ ${ }^{4}$ Lin Gao County People's Hospital of Hainan Province, Haikou, China \\ Email: ^g2002m@163.com
}

How to cite this paper: Guo, J.C., Guo, M., Huang, P., Yang, Y.J., Jiang, X.L. and Cen, W.D. (2019) Correlation between Serotonin Transporter Promoter Gene Polymorphism and PTSD in Children. International Journal of Clinical Medicine, 10, 336-344. https://doi.org/10.4236/ijcm.2019.105026

Received: May 5, 2019

Accepted: May 27, 2019

Published: May 30, 2019

Copyright (c) 2019 by author(s) and Scientific Research Publishing Inc. This work is licensed under the Creative Commons Attribution International License (CC BY 4.0).

http://creativecommons.org/licenses/by/4.0/

\section{Open Access}

\begin{abstract}
Objective: To study the association between post-traumatic stress disorder (PTSD) and serotonin transporter promoter (5-HTTLPR) gene polymorphism in Han children in Hainan; to explore the genetic mechanism of PTSD in children. Methods: 50 patients with post-traumatic stress disorder and healthy children in Han nationality in Hainan were selected. Detection of 5-HTTLPR gene polymorphism by polymerase chain reaction (PCR) and amplified fragment length polymorphism, the genotype and allele frequencies were analyzed using a case-control association analysis method. Results: There were 4, 14 and 32 cases of LL, SL and SS in the post-traumatic stress disorder group of Hainan Han children, and 13, 20 and 17 cases in the control group. From the perspective of gene frequency, the L gene of post-traumatic stress disorder appeared $22.0 \%$, and S appeared $78.0 \%$. In the control group, L appeared $46.0 \%$, and S appeared 54.0\%. There were significant differences in genotype and gene frequency $(\mathrm{P}<0.01)$. Conclusion: The 5-HTTLPR gene polymorphism in Hainan Han children may be associated with post-traumatic stress disorder.
\end{abstract}

\section{Keywords}

Post-Traumatic Stress Disorder, Genetic Polymorphism, A Serotonin Transporter Promoter, Genetics

\section{Introduction}

Children in the fields of psychiatry, pediatrics, child care and education have 
conducted extensive research on the causes of children with PTSD, involved in various fields such as genetics, immunology, virology, neurobiology and psychosocial. Among them, the research on genetics has become an important research direction in the current psychiatric community [1]. In recent years, natural disasters and man-made disasters have occurred frequently, and the incidence of post-traumatic stress disorder (PTSD) in children has increased significantly [2]. The main symptoms of PTSD are: repeated traumatic experience (flashback), traumatic situations involuntarily emerged; second, continuous avoidance and stimulation related to traumatic events; third, sustained alertness [3]. Therefore, exploring the mechanism of occurrence of PTSD in children is very necessary for its prevention and treatment. The serotonin transporter (5-HTTLPR) is distributed on the presynaptic membrane of 5-HT neurons, which plays an important role in the reabsorption of 5-HT neurotransmitters in the synaptic cleft, 5-HT system functional status [4]. In recent years, studies have shown that dysfunction of the serotonin system plays a role in the development of children with PTSD. Therefore, the 5-HTTLPR gene is an important candidate gene for PTSD in children [5]. This study also further explored the correlation between the genotype and allele frequency of 5-HTTLPR in the PTSD group and the control group of Han children in Hainan. It provides a scientific basis for the clinical diagnosis of children with PTSD and provides a scientific basis for the genetics of PTSD in children.

\section{Objects and Methods}

\subsection{Object}

All cases were from December 2016 to December 2017. The investigation was conducted in the psychological clinic of Lin Gao County People's Hospital of Hainan Province and Hainan Provincial People's Hospital for the diagnosis of PTSD Han children. The PTSD Diagnostic Scale and the diagnostic criteria for PTSD in the US Diagnostic and Statistical Manual of Mental Disorders (DSM-IV) have been used.

Inclusion criteria: 1 age is 4 to 12 years old; 2 right hand; 3 Comply with the diagnostic criteria for PTSD in the US Diagnostic and Statistical Manual of Mental Disorders (DSM-IV). Exclusion criteria: Associated with mental retardation, personality disorder, material dependence or alcohol dependence. Volunteer to participate in the study and to obtain informed consent (child or his father, mother). The study group consisted of 26 males and 24 females with an average age of $6.26 \pm 2.40$ years. The control group consisted of 27 males and 23 females with an average age of $5.92 \pm 2.98$ years. There was no significant difference in gender and age between the two groups $(\mathrm{P}>0.05)$.

\subsection{Methods}

Genomic DNA was extracted by a conventional phenol chloride method. The PCR primers are as follows: positive 5'-GGCGTTGCCGCTCTGAATGC-3, Re- 
verse 5'-GAGGGACTGAGCTGGACAACCAC-3, using TaKaRaEx from Bio-Engineering (Dalian) Co., Ltd. The PCR product was subjected to vertical electrophoresis on a $6 \%$ non-denaturing polyacrylamide gel. After ethidium bromide (EB) staining, the electrophoresis images of each sample were detected by an ultraviolet gel analyzer to determine the genotype.

\subsection{Statistical Analysis}

Data analysis was performed using SPSS 13.0 statistical software. Methods include: Hardy-Weinberg (H-W) coincidence test, frequency analysis, analysis of variance, $\mathrm{t}$ test, and $\chi^{2}$ test.

\section{Results}

There was no significant difference in the sex ratio, age and education between the study group and the control group ( $\mathrm{P}>0.05$, Table 1$)$.

Hardy-Weinberg's Law of Genetic Balance Test. The Hardy-Weinberg's law of genetic equilibrium tests that the gene distribution of 5-HTTLPR conforms to the law of genetic balance and is representative of the population (Table 2).

Compared with the genotype and allele of the PTSD group and the control group, the 5-HTTLPR gene showed 4, 14, and 32 cases of LL, SL, and SS in the post-traumatic stress disorder group, respectively, and 13, 20, and 17 cases in the control group. From the perspective of gene frequency, the $\mathrm{L}$ gene of post-traumatic stress disorder appeared $22.0 \%$, and $S$ appeared $78.0 \%$. In the control group, L appeared $46.0 \%$, and S appeared $54.0 \%$, there were significant differences in genotype and gene frequency between the two groups $(\mathrm{P}<0.01$, Table 3).

Table 1. Comparison of basic situation between PISD group and control group.

\begin{tabular}{|c|c|c|c|}
\hline \multirow{2}{*}{ Group } & \multicolumn{2}{|c|}{ gender } & \multirow[t]{2}{*}{ Age (year old) } \\
\hline & male & female & \\
\hline PTSD group & 26 & 24 & $6.26 \pm 2.40$ \\
\hline Control group & 27 & 23 & $5.92 \pm 2.98$ \\
\hline$X^{2}$ or $t$ & \multicolumn{2}{|c|}{0.067} & 0.487 \\
\hline$P$ & \multicolumn{2}{|c|}{0.795} & 0.314 \\
\hline
\end{tabular}

- by $X^{2}$ test $\boldsymbol{\Delta}$ by t test. The healthy control group is a hospital-school joint health check-up child, which is randomly enrolled, and there is no significant difference in gender, age, etc.

Table 2. 5-HTTLPR gene distribution Hardy-wcinbcrg genetic equilibrium law test.

\begin{tabular}{cccccc}
\hline & \multicolumn{3}{c}{ genotype } & & \\
\cline { 2 - 4 } Group & LL & SL & SS & $X^{2}$ & \\
\hline $\begin{array}{c}\text { PTSD } \\
\text { group }\end{array}$ & 4 & 14 & 32 & 1.696 & 0.193 \\
$\begin{array}{c}\text { Control } \\
\text { group }\end{array}$ & 13 & 20 & 17 & 1.899 & 0.168 \\
\hline
\end{tabular}


Table 3. Comparison of genotypes and alleles between PISD group and control group (n $=50, \%)$.

\begin{tabular}{cccccc}
\hline \multirow{2}{*}{ Group } & \multicolumn{3}{c}{ Genotype frequency (\%) } & \multicolumn{3}{c}{ Allele frequency (\%) } \\
\cline { 2 - 6 } & LL & SL & SS & L & S \\
\hline $\begin{array}{c}\text { PTSD } \\
\text { group }\end{array}$ & $4(8.0)$ & $14(28.0)$ & $32(64.0)$ & $22(22.0)$ & $78(78.0)$ \\
$\begin{array}{c}\text { Control } \\
\text { group }\end{array}$ & $13(26.0)$ & $20(40.0)$ & $17(34.0)$ & $46(46.0)$ & \\
$X^{2}$ & 10.415 & & & 12.834 & \\
$P$ & 0.017 & & & 0.0002 & \\
\hline
\end{tabular}

\section{Discussion}

With the rapid development of modern society, the radius of children's activities has expanded, and the use of various vehicles has become more and more frequent. The acute trauma caused by traffic accidents has gradually become a risk factor threatening people's lives and health [6]. A car accident (or witness to a car accident) as a serious, sudden stressful life event can lead to a strong psychological and physical dual stress response in the injured [7]. The stress response caused by this unconventional emergency causes the injured person to cause different degrees of physiological damage on the body, and also causes a series of physiological, psychological and behavioral changes and degrees under the action of the traumatic stress source. Different psychological stress states [8]. A car accident is sudden, unbearable or catastrophic. The physical and psychological impact on the patient is very direct and significant, and often produces a strong psychological stress response, affecting the treatment effect and prognosis.

Post-traumatic stress disorder is a delayed and long-lasting mental disorder caused by unusual, threatening psychological trauma. Specific symptoms of PTSD include re-experience, avoidance, and increased alertness following traumatic events. The diagnostic criteria must include a specific number of each of the three types of symptoms: re-experience, avoidance, and alertness. A large number of studies have confirmed that [9] children with PTSD have obvious family aggregation and genetic predisposition. With the rapid development of molecular genetics and molecular biology technology, many susceptibility genes related to the pathogenesis of PTSD have been identified. Among them, the HTSD gene is closely related to PTSD, but the research conclusions are not the same. Studies have shown that [10], The TDT analysis of the PTSD core family found that the 5-HTT promoter region (5-HTTLPR) S allele was preferentially transmitted to the children, and the 5-HTT gene intron VNTR polymorphism was not significantly associated with PTSD; Two-point haploid TDT analysis showed significant differences in 5-HTTLPR and VNTR allele transmission, and the study found that the [11] L allele was preferentially transmitted to children, The core family TDT study further found no difference in 5-HTTLPR allele 
transmission, but was related to the severity of social disorders, in the severely disordered individuals, the $\mathrm{S}$ allele is preferentially transmitted, and $\mathrm{L}$ allele delivery is superior in individuals with mild to moderate disease, suggesting that the 5-HTTLPR allele itself does not determine the susceptibility of PTSD, which may be related to the severity of social disorders. Cao Yuping et al. [12] reported the transmission imbalance of 5-HTTLPR and the second intron VNTR haplotype, but 5-HTTLPR as an independent marker did not have a transmission imbalance. In summary, the 5-HTT gene remains a candidate gene for PTSD. Studies have shown that 5-HTR2A is highly densely distributed in the frontal cortex, and the frontal cortex is closely related to cognitive function, suggesting that 5-HTR2A may be the main receptor link in children with autism 5-HTT neurological abnormalities. It is hypothesized that [13], individual differences in susceptibility to PTSD in children may be related to differences in 5-HTR2A function and expression levels in the brain, whereas differences in 5-HT2A function and expression levels are determined by 5-HTR2A gene polymorphisms.

There are many studies on PTSD and its pathogenesis, but the influence of genetic factors is still unclear. The genetic research of PTSD is still in its infancy, and the number of studies is small, and it is mostly in the polymorphic analysis of single genes and single sites. Because PTSD itself is highly heterogeneous in clinical practice, it should be combined with epigenetics and traditional genetics to explore its pathogenesis from the interaction between genetic susceptibility and environment of PTSD. The mechanism of 5-HT system in PTSD pathology is unclear, but 5-HT is thought to be associated with PTSD's mood swings, irritability, and sleep abnormalities [14]. The 5-HT transporter gene (5-HTT gene) is one of the most widely studied genes involved in increased 5-HT activity following stress events. Abnormal 5-HT activity may mediate emotional abnormalities. Susceptibility to PTSD [15]. Therefore, regulation of the 5-HT system gene may play a role in the depression response of various stressors in the susceptibility or life process of PTSD. Allelic variation in human 5-HTT expression is due to a 5-HTTLPR polymorphism in the promoter region containing two major alleles, which are long alleles (L) and short Gene (S). In vitro experiments showed that long fragment $\mathrm{L}$ had higher transcriptional activity than short fragment $\mathrm{S}$. Brain tissue and cultured cells with LL genotype produced 5-HTT mRNA and protein expression were higher than SS genotype, Therefore, 5-HTTLPR may affect the serotonin system function by affecting the transcriptional activity of the gene and the function of the 5-HTT protein, thereby affecting the individual's emotional, mental, and personality disorders [16] [17]. Annette $\mathrm{M}$ et al. [18] analyzed the 5-HTTLPR gene in patients with PTSD and healthy subjects and found that the SS genotype of patients with PTSD was significantly higher than that of healthy controls. Fernando et al. [19] found that the $S$ allele in the 5-HTTLPR polymorphism significantly increased the risk of PTSD in hurricane-affected populations. The highest risk was SS genotype, low social support, and severe hurricane trauma. A large number of previous studies have shown that 5-HTTLPR is associated with many mental illnesses, such as SS genotypes in 
patients with severe depression and anxiety [20] [21] [22]. A large sample of twin studies showed that 5-HTTLPR individuals with SS or SL were prone to PTSD depressive responses after stress events, and further analysis revealed 5-HTT gene polymorphism and post-stress affective disorder. The occurrence is related [23]. Other studies have shown that SS or SL allele carriers have higher amygdala activity than LL alleles when they encounter fear stimulation, while SL carriers have lower anxiety than LL, suggesting that fear and anxiety may be related to 5-HTT gene mutation [24]. Some studies have shown that individuals with a 5-HTT polymorphism with two short alleles (SS or SL) are more sensitive to stress than individuals with only one or two long alleles (LL) [25]. In conclusion, 5-HTT may play a role in the degree of stress response, and its gene mutation can regulate the depression sensitivity of individuals to stress. Although there are few studies on the susceptibility of 5-HTT and PTSD, however, the increase in amygdala activity caused by fear stimulation is more likely to occur in individuals carrying the homozygous SS gene or the heterozygous SL gene than the homozygous LL gene.

We analyzed the 5-HTTLPR gene polymorphism in 50 children with PTSD and 50 healthy children in Hainan, and found that there were significant differences in genotype and gene frequency. This indicates that the 5-HTTLPR gene polymorphism is associated with pathological and behavioral changes under stress. Therefore, it can be shown that the 5-HTTLPR gene polymorphism is associated with PTSD. The sequencing results of this study showed that the proportion of $S$ allele was significantly higher than that of $\mathrm{L}$ allele $(\mathrm{S}=78.0 \%$; $\mathrm{L}=$ $22.0 \%$ ), and genotype SS and LS alleles were dominant, this is basically consistent with the results of Asian populations such as China ( $\mathrm{S}=77.0 \% ; \mathrm{L}=23.0 \%)$ and Japan $(\mathrm{S}=82.3 \% ; \mathrm{L}=17.7 \%)$, However, there are differences between European and North American populations. Among these populations, allele L ( $\mathrm{S}=$ 45.3\%; $\mathrm{L}=54.7 \%)$ and genotype LL account for a high proportion [26]. This series of differences considers racial heterogeneity, so ethnic background effects need to be considered in the 5-HTTLPR polymorphism study [27]. For the relationship between PTSD and 5-HTTLPR polymorphism, there are related studies abroad, but there is no clear report on the Chinese population. This study can clarify that PSTD and 5-HTTLPR are also related in the Han population of Hainan Province.

\section{Conclusion}

Post-traumatic stress disorder is an important manifestation of mental and behavioral disorders after unconventional emergencies. It has the characteristics of high incidence, high prevalence, long course of disease and poor curative effect, which seriously affects clinical treatment. At present, domestic and international research on the internal relationship between 5-HT system and children's PTSD is of great concern. It is helpful to clarify the etiology and pathogenesis, and may have positive significance for diagnosis, treatment and prevention. 


\section{Fund-Funded Projects}

2017 Hainan Natural Science Foundation No.: 817405.

\section{Conflicts of Interest}

The authors declare no conflicts of interest regarding the publication of this paper.

\section{References}

[1] Geronazzo-Alman, L., Fan, B., Duarte, C.S., et al. (2019) The Distinctiveness of Grief, Depression, and Posttraumatic Stress: Lessons from Children after 9/11. Journal of the American Academy of Child and Adolescent Psychiatry. https://doi.org/10.1016/j.jaac.2018.12.012

[2] Silwal, S., et al. (2019) Parental Immigration and Offspring Post-Traumatic Stress Disorder: A Nationwide Population-Based Register Study. Journal of Affective Disorders, 249, 294-300. https://doi.org/10.1016/j.jad.2019.02.002

[3] Fredman, S.J., et al. (2019) Parents' PTSD Symptoms and Child Abuse Potential during the Perinatal Period: Direct Associations and Mediation via Relationship Conflict. Child Abuse \& Neglect, 90, 66-75.

https://doi.org/10.1016/j.chiabu.2019.01.024

[4] Carmassi, M., Corsi, C.A., Bertelloni, V., Pedrinelli, G., et al. (2019) Post-Traumatic Stress and Major Depressive Disorders in Parent Caregivers of Children with a Chronic Disorder. Psychiatry Research. https://doi.org/10.1016/j.psychres.2019.02.062

[5] Auxéméry, Y. (2012) Posttraumatic Stress Disorder (PTSD) as a Consequence of the Interaction between an Individual Genetic Susceptibility, a Traumatogenic Event and a Social Context. L'Encephale, 38, 373-380.

[6] Agorastos, A., et al. (2019) Relations of Combat Stress and Posttraumatic Stress Disorder to 24-h Plasma and Cerebrospinal Fluid Interleukin-6 Levels and Circadian Rhythmicity. Psychoneuroendocrinology, 100, 237-245.

https://doi.org/10.1016/j.psyneuen.2018.09.009

[7] Motz, T.A. and Currie, C.L. (2019) Racially-Motivated Housing Discrimination Experienced by Indigenous Postsecondary Students in Canada: Impacts on PTSD Symptomology and Perceptions of University Stress. Public Health. https://doi.org/10.1016/j.puhe.2018.12.011

[8] Nelson, S., Conroy, C. and Logan, D. (2019) The Biopsychosocial Model of Pain in the Context of Pediatric Burn Injuries. European Journal of Pain (London, England), 23, 421-434. https://doi.org/10.1002/ejp.1319

[9] Karam, E.G., et al. (2019) Role of Childhood Adversities and Environmental Sensitivity in the Development of Post-Traumatic Stress Disorder in War-Exposed Syrian Refugee Children and Adolescents. The British Journal of Psychiatry. The Journal of Mental Science, 214, 354-360.

[10] Xie, P., Kranzler, H.R., Poling, J., Stein, M.B., et al. (2009) Interactive Effect of Stressful Life Events and the Serotonin Transporter 5-HTTLPR Genotype on Posttraumatic Stress Disorder Diagnosis in 2 Independent Populations. Archives of General Psychiatry, 66, 1201-1209.

https://doi.org/10.1001/archgenpsychiatry.2009.153

[11] Walsh, K., et al. (2014) Associations between the SS Variant of 5-HTTLPR and 
PTSD among Adults with Histories of Childhood Emotional Abuse: Results from Two African American Independent Samples. Journal of Affective Disorders, 161, 91-96. https://doi.org/10.1016/j.jad.2014.02.043

[12] Smolka, M.N., et al. (2019) Smoking Moderates Association of 5-HTTLPR and in Vivo Availability of Serotonin Transporters. European Neuropsychopharmacology: The Journal of the European College of Neuropsychopharmacology, 29, 171-178.

[13] Xie, P., et al. (2009) Interactive Effect of Stressful Life Events and the Serotonin Transporter 5-HTTLPR Genotype on Posttraumatic Stress Disorder Diagnosis in 2 Independent Populations. Archives of General Psychiatry, 66, 1201-1209. https://doi.org/10.1001/archgenpsychiatry.2009.153

[14] La Greca, A.M., et al. (2013) Children's Risk and Resilience Following a Natural Disaster: Genetic Vulnerability, Posttraumatic Stress, and Depression. Journal of Affective Disorders, 151, 860-867. https://doi.org/10.1016/j.jad.2013.07.024

[15] Shan, L., Guo, H.-Y., Corina, N.A.M., et al. (2018) Impaired Fear Extinction in Serotonin Transporter Knockout Rats Is Associated with Increased 5-Hydroxymethylcytosine in the Amygdala. CNS Neuroscience \& Therapeutics, 24, 810-819. https://doi.org/10.1111/cns.12822

[16] Schipper, P., Henckens, M.J.A.G., et al. (2018) Acute Inescapable Stress Alleviates fear Extinction Recall Deficits Caused by Serotonin Transporter Abolishment. Behavioural Brain Research, 346, 16-20. https://doi.org/10.1016/j.bbr.2017.12.009

[17] Liu, L., et al. (2018) Serotonin Transporter 5-HTTLPR Genotype Is Associated with Intrusion and Avoidance Symptoms of DSM-5 Posttraumatic Stress Disorder (PTSD) in Chinese Earthquake Survivors. Anxiety, Stress, and Coping, 31, 318-327. https://doi.org/10.1080/10615806.2017.1420174

[18] Laplante, D.P., Simcock, G., et al. (2018) The 5-HTTLPR Polymorphism of the Serotonin Transporter Gene and Child's Sex Moderate the Relationship between Disaster-Related Prenatal Maternal Stress and Autism Spectrum Disorder Traits: The QF2011 Queensland Flood Study. Development and Psychopathology, 1-15.

[19] Navarro-Mateu, F., et al. (2013) Meta-Analyses of the 5-HTTLPR Polymorphisms and Post-Traumatic Stress Disorder. PLoS ONE, 8, e66227.

https://doi.org/10.1371/journal.pone.0066227

[20] Bryant, R.A., et al. (2010) Preliminary Evidence of the Short Allele of the Serotonin Transporter Gene Predicting Poor Response to Cognitive Behavior Therapy in Posttraumatic Stress Disorder. Biological Psychiatry, 67, 1217-1219. https://doi.org/10.1016/j.biopsych.2010.03.016

[21] Gressier, F., et al. (2013) The 5-HTTLPR Polymorphism and Posttraumatic Stress Disorder: A Meta-Analysis. Journal of Traumatic Stress, 26, 645-653.

https://doi.org/10.1002/jts.21855

[22] Luis, J.-T., et al. (2019) 5-HTTLPR-Brain-Derived Neurotrophic Factor (BDNF) Gene Interactions and Early Adverse Life Events Effect on iMpulsivity in Suicide Attempters. The World Journal of Biological Psychiatry: The Official Journal of the World Federation of Societies of Biological Psychiatry, 20, 137-149.

[23] Grabe, H.J., et al. (2009) Serotonin Transporter Gene (SLC6A4) Promoter Polymorphisms and the Susceptibility to Posttraumatic Stress Disorder in the General Population. The American Journal of Psychiatry, 166, 926-933.

[24] Grabe, H.J., et al. (2012) Moderation of Adult Depression by the Serotonin Transporter Promoter Variant (5-HTTLPR), Childhood Abuse and Adult Traumatic Events in a General Population Sample. American Journal of Medical Genetics. Part $B$, Neuropsychiatric Genetics. The Official Publication of the International Society 
of Psychiatric Genetics, 159B, 298-309. https://doi.org/10.1002/ajmg.b.32027

[25] Koenen, K.C., et al. (2009) Modification of the Association between Serotonin Transporter Genotype and Risk of Posttraumatic Stress Disorder in Adults by County-Level Social Environment. American Journal of Epidemiology, 169, 704-711. https://doi.org/10.1093/aje/kwn397

[26] Zhang, Y., Ming, Q.-S., Yi, J.-Y., et al. (2017) Gene-Gene-Environment Interactions of Serotonin Transporter, Monoamine Oxidase A and Childhood Maltreatment Predict Aggressive Behavior in Chinese Adolescents. Frontiers in Behavioral Neuroscience, $11,17$.

[27] Grabe, H.J., Spitzer, C. and Schwahn, C. (2009) Serotonin Transporter Gene (SLC6A4) Promoter Polymorphisms and the Susceptibility to Posttraumatic Stress Disorder in the General Population. American Journal of Psychiatry, 166, 926-933. https://doi.org/10.1176/appi.ajp.2009.08101542 